\title{
Research on the Construction of Digital Sports Campus in Colleges
}

\author{
Leilei $\operatorname{Tian}^{1}$ and Cunjun $\mathrm{Xie}^{2}$ \\ ${ }^{1}$ Jingdezhen Ceramic University, Jingdezhen, Jiangxi 333403; \\ ${ }^{2}$ Jingdezhen College, Jingdezhen, Jiangxi 333000
}

\begin{abstract}
The concept of digital sports is defined by using the literature method and the field investigation method. The present situation of the digital sports campus construction is as follows: 1 . The digitalization of the physical and mental fitness of the teachers and students in the university; 2. The digitalization of the equipment; 3 . The digitization of the content and the teaching process of the sports course; 4. The digitization of student physical fitness test evaluation. Campus digital construction still have many problems, the performance is that the APP docking of digital products lack management platform, 2 the shortage of digital sports talent; 3 the digital test equipment for student physical testing should be innovative, evaluation needs more comprehensive. It is concluded that the development of digital sports campus in the background of scientific and technological development depends on the personnel training of digital sports talents and the development of digital hardware and software to solve the problems brought by the digitalization of sports and promote the development of digitalization of campus sports.
\end{abstract}

Keywords: College physical education; Digital sports; Jiangxi colleges

\section{高校数字化体育校园建设的研究}

\author{
田蕾蕾 ${ }^{1}$, 谢存军 ${ }^{2}$ \\ （1.景德镇陶瓷大学，江西 景德镇 333403 2. 景德镇学院体育系，江西 景德镇 333000 )
}

摘要: 运用文献资料法、实地调查法, 对数字化体育的概念进行界定, 数字化体育校园建设的现状表现为 1 高校师生运 动健身数字化; 2 场地器材数字化; 3 体育课程内容和教学过程数字化; 4 学生体质测试评价数字化。校园数字化建设还存 在较多问题, 表现在 1 数字化产品 APP 对接缺少管理平台, 2 数字化体育人才短缺; 3 学生体质测试数字化测试仪器应创新, 评价需要更加全面化。得出结论: 科技大发展背景下数字化体育校园的发展, 有赖于专业体育数字化人才的培养和数字化硬 件和软件的开发, 解决体育数字化带来的问题, 促进校园体育数字化的发展。

关键词: 高校体育; 数字化体育; 江西高校

中图分类号: G807 文摘标识码: A

引言

进入 21 世纪, 科学技术变化日新月异, 特别是电子信息科技的进步引领了信息时代的到来 ${ }^{[1]}$, 信息的 数字化可以说是引领世界的第三次科技革命, 信息的数字化对各行各业产生了积极的影响。教育行业首先 受数字化的推动, 其中体育数字化教育也紧跟步伐。体育教育的传统概念的受到影响和冲击, 表现在体育 教育的观念、内容、方法手段、格局和规模等。转变传统的思维观念, 对数字化产品加以应用, 合理服务 体育教育是整个江西甚至全国高校所面临的新问题。江西高校应紧跟时代需求, 在基于社会发展和学校体 育管理需要的基础上, 进行数字化体育校园的建构 ${ }^{[2]}$ 。

\section{1 高校数字体育概念的界定}

本文研究的高校数字体育, 即数字校园体育 (Digitla Campus Physical Education 简 称 DCPE) 是 指以互联网为基础, 充分利用先进的信息化平台和工具, 实现从体育教学和锻炼环境（包括多媒体体育教 
学设备、场馆、教室等)、资源（如体育图书、讲义、课件等）到活动（包括教、学、管理、服务、办公、 竞赛等) 的全部体育数字化, 在传统体育范畴的基础上构建一个数字空间, 以拓展体育资源的时间和空间 维度, 提升传统体育的服务效率, 扩展传统体育的功能, 最终实现体育教学和锻炼过程的全面数字化。从 而达到提高体育教学管理水平和效率的目的。

\section{2 高校数字化发展现状}

数字化校园建设有其发展的多个方面, 主要体现在体育资源数字化、学生教师锻炼健身数字化、体育 教学数字化、学生体质测试数字化、体育管理数字化等。

\section{1 校园体育场地器材数字化}

校园内部的数字化体育设施也越来越普遍。多媒体教室已经由普通多媒体教室引进到了体育场馆内 部。体育场馆内的 LED 大小屏幕越来越常见, 首先看到的是场馆的使用安排表, 教师上课安排表等, 学生 和教师可以很清楚的看到正在上课和使用场馆的教师和教学项目, 以及该周场馆的安排使用状况, 甚至场 馆管理者的使用联系方式及值班人员信息。场馆内部的 LED 大屏幕则是可以与场馆的比赛, 活动背景直接 相关。特别是比赛, 更能够直观看到场上比赛的状况。学校体育数字化设施还不能和职业比赛相比, 究其 原因可能是学校场地设施的投入经费有限, 该高校没有承担更高标准的多功能的场馆的需要, 这在在不同 高校有不同的表现, 特别是地方高校对综合场馆的运用多数是校内使用, 主要是满足教学和校园活动。大 城市的综合性高校则有承担体育竞赛的需要数字化水平较高, 特别体现在座位和对应的座位票可以数字化 管理。

\section{2 高校师生运动健身数字化}

随着智能手机和 APP (英文 Application 的简称, 中文意思为应用软件) 结合的全面升级, 数字化体 育在校园的流行越来越贴近生活。高校大学生对健身的需求以及对自己健康的管理, 促使学生使用新生代 高智商的大学生, 首先试用新的电子产, 在全校学生和老师都在试用智能手机的数字化背景下, APP 也把 有关运动测试的软件研发并投入使用。如苹果新出的产品 Apple Watch, 所具有的功能有体能训练 App, 从骑车、跑步等种种运动中获取详细的测量数据, 使用者可以直观的了解自身的锻炼状况 ${ }^{[3]}$ 。国内产品中, 小米系列也推出了众多数字体育 $\mathrm{APP}$, 如小米手环 ${ }^{[4]}$ 与手机结合, 可以为使用者分析运动睡眠数据, 提供 健康建议, 具体包括运动总步数, 公里数, 消耗的卡路里值, 睡眠的时间和质量等。具实际调查了解学生 的使用运动 APP 的状况, 发现使用率正在逐步提高。高校可以充分利用这些资源来深入了解学生的锻炼状 况, 监测学生的体质健康水平。

\section{3 体育课程内容及过程数字化}

体育课程数字化的应用主要体现在专业体育教室数字化: 连接投影仪或者数码电子屏幕的高倍摄像 机, 从不同的角度拍摄学生的学习动作技能, 并在专业教师讲解中予以回放, 使学生更加直观的看到自己 的动作技能的不足, 教学中教师通过对所录视频的慢动作的回放, 可以更加直观的给学生以指导。在对一 些相关视频的技术套路的学习中, 通过对原有视频的慢放、分段放、重复放等, 使学生更加清楚每个技术 动作的规范性，使用的数码显示屏或者 LED 大屏幕，可以更加容易看清技术动作细节。

多媒体技术已经发展十余年, 从技术到应用以基本趋于成熟, 教师准备的教学课件, 通过网络共享, 学生随时可以下载学习。包括网络数据库在内的数据资源, 使学生的上课不在拘泥于教室内, 而是在有网 
络的任何地方。特别是有全校有Wifi 覆盖的高校, 只要有可以连接的无线网络的电脑或手机, 都可以在 线进行学习。有条件的高校已经着手建设体育网络课程, 把全校各体育项目的精品课程制成网络数据库, 学生可以尽情享受与学校环境相适应的网络体育学习, 甚至可以实现与教师的对话, 以及学生疑问的解答, 教师进行问题汇总后可以集中进行现场教学、引导、纠错等, 为学生解决实际的问题。

在智力性运动中, 智力 APP 的引入, 教室内学生使用网络连接教师的电脑, 可以全面了解教师的操作 和讲解, 如桥牌的新睿桥牌 APP, 就可以建立群, 不同的学生打同一副牌, 可以产生不同的结果, 教师可 以随时予以讲解，使学生更加生动形象的明白打法以及学生之间的相互交流。

\section{4 学生体质测试评价数字化}

国家教育部针对学生的体质健康水平所要求的学生体质健康测试, 每年都要测试一次, 并通过网络系 统上报, 在高校体育主管部门都有这样一套测试设施和上报系统。通过测试设施的实际操作测试, 与之相 匹配的系统存储并汇总分析每个学生的测试结果, 在测试的结果中, 可以查到学生的每一项测试成绩, 并 给出综合评定的分数以及所属成绩的类别 (优、良、中不合格)。如今各种仪器通过研发, 有了新的进展, 如比较费时费力的仰卧起测试, 研发出了仰卧起坐仪, 男生的引体向上测试, 研发出了引体向上测试仪。 仪器配有芯片和软件, 能自动识别所做的规范性和次数, 并能够记录次数。各种仪器和软件的应用已经是 学校的测试减少了工作量、降低的工作的强度, 提高了工作效率。在评价中, 数据库的建立, 使得对测试 数据马上得出一个评价。

\section{5 体育行政管理的数字化}

现代网络交流平台的建设和发展, 促使行政管理越来越方便, 体育的行政管理也在一步步实现数字化。 体育行政管理中体育教师、体育课程、所有参与大学生体育健康课程的普通学生和体育专业学生、以及体 育管理方面的人员, 分门别类的数字化管理, 使得行政管理越来越精细化和程序化, 而因此出现的差错则 越来越少。在有关体育师资的管理中, 体育教师的档案数字化、科研教学量数字化、并根据每个人的课时、 科研量来给予每位体育教师公平合理的评价。在评优评先中得以明确和具体，通过网络平台的共享，每个 人可以公开的信息则被所有同行认可。学生数字化管理则体现在学生的健身档案数字化, 学生可以通过网 络查询到自己的体育成绩、体质测试成绩，具体在全校的排名，自身某些方面的优势和不足系统给予一个 综合的评价。系统的管理人员通过不断更新数据，使得整个体育行政管理的时效性得以体现。

\section{3 高校数字体育数字化在发展的问题}

在体育数字化加速的大环境下, 高校体育数字化还存在很多不足。校园数字化体育设施有待进一步研 发和更新, 新兴运动 APP 缺少资源整合和利用等, 具体表现在以下方面: 数字化体育人才缺少, 体育数字 化的产品 APP 缺少管理对接平台, 学生体质测试数字化测试仪器应创新, 评价需要更加全面化, 数字化体 育管理需更加广泛和深入等。

\section{1 数字化产品 APP 对接缺少管理平台}

目前, 数字化产品随着手机等电器软件的升级, 学校体育对接平台还没有建立, 对学生日常活动缺少 有效的了解及跟踪评价, 学生的体育课活动、课外体育活动, 都可以由学生使用的手环或手表进行对接了 解。学生上课活动的多少, 测试成绩的高低, 均可得到有效的数据, 在对学生体育课上课质量和老师上课 效果上都可给予量化的评价。课外体育活动是学生在学校内外参与的有利于自身健康的体育运动, 是学生 
在促进身体健康主动运动的良好体现, 通过软件的对接, 了解学生课外体育活动的频率、强度、健康促进 的水平，则降低了学生的体质测试成绩因状态和其他原因测试不准确的概率。

\section{2 数字化体育人才短缺}

唐瑞民曾提出, 数字化体育人才缺乏是制约体育领域数字化的关键问题之一, 不培养体育数字化人才, 将严重影响体育数字化前进的步伐 ${ }^{[5]}$ 。通过数字体育专业人才, 可以架设起体育与信息技术之间的桥梁, 促进体育数字化的发展 ${ }^{\left[{ }^{[6]}\right.}$ 。数字化体育人才的缺少, 源于几乎所有高校没有专门培养这方面的人才。既要 懂的体育专业知识, 又要精通计算机、电子方面的知识, 就研发体育数字化产品是一般人做不到的。这也 是现代体育科研方面为什么总出现其他学科与体育学科相互合作的科研团队出现的原因。因此, 高校在对 信息工程或者电子方面专业学生的培养时, 可以增加学生对体育专业知识的教授, 促进这些学生既不脱离 自己的专业, 有能拓展对体育领域的研究; 也可对体育专业的学生进行数字化教育, 通过对信息技术的学 习, 扎实多方面专业知识, 使之成为能够为体育数字化发展的可用人才。

\section{3 学生体质测试数字化测试仪器应创新, 评价需要更加全面化}

每年由国家教育部规定的学生体质测试，通过学生体质健康网进行上报，数据之大，测试繁琐已是不 争的事实。在体育数字化进程加速发展的当下, 学生体质测试的仪器有待进一步更新。如测试学生的 $50 \mathrm{~m}$ 、 $800 \mathrm{~m}$ (女) $/ 1000 \mathrm{~m}$ (男), 手计时和电计时同样适用, 先比之下的手计时早应被电计时取代, 但是, 多数 高校的电计时仪器和系统并没有与时俱进。仰卧起坐（女）和引体向上（男), 坐位体前屈等指标, 虽然 已经有了测试仪, 但仪器相对固定, 如每个人都配有智能测定的运动手环, 通过电脑或手机的 APP 了解个 人的锻炼状况和达标水平, 因此, 更智能数字化仪器和系统的研发相对缺少。

对学生体质健康标准的评价也需要更加全面和深化, 一次的测试成绩不能代表学生的最好水平, 仅以 一次的成绩来评价学生缺少客观性, 如果学生能够定期自主测定, 则用其多次测试的成绩来反映该学生的 健康水平, 会明显增加其成绩的客观性。针对测试的某一项偏差的指标, 学生可根据实际情况对该方面进 行有效的锻炼, 以此提高整体成绩。

若有条件，学生每人可佩戴一个手环一样的仪器，学校建立一个系统平台，只要佩戴上该设备既可以 监督学生的锻炼状况, 也可进行多项指标的测试, 排除人为记录的差错, 系统通过梳理把测试或监督的数 据给学生, 以此让学生能够及时了解自身的锻炼状况和体质健康水平。如今, 既没有这样仪器设备, 也没 有这样的管理平台, 评价具有很大的偶然性, 因此就学生体质健康标准的测试和评价和有很长的路要走。

\section{4 小结}

高校体育数字化进程中，进步和成绩已为大多数人认可。但在高校体育数字化的背景下，人才对数字 化的贡献举足轻重, 但专业化人才的培养还未得到有关部门的重视。科学技术快速发展带来了新兴的仪器 和 APP, 但作为学校和高级教育主管部门, 并没有及时更新思维, 重视学生对新仪器和 APP 的应用, 并建 立相应的网络平台与之对接, 以此来了解学生的身体锻炼状况和体质健康水平, 给予客观合理的评价。

\section{致谢}

基金项目:景德镇陶瓷大学青年基金课题《江西数字体育发展规划初探》（2015-06）

\section{Acknowledgement}

Fund Project: Jingdezhen Ceramic University Youth Fund Project "Jiangxi Digital Sports Development Plan" 
(2015-06)

\section{参考文献}

[1] (美) 尼葛洛庞帝著, 胡泳, 范海燕译. 数字化生存 [M].海南: 海南出版社, 1997 .

[2]王力晨. “数字校园体育” 的建构 [J]. 沈阳体育学院学报, 2003, 03:20-22.

[3]http://www. apple. com/cn/watch/

[4]http://www. mi. com/shouhuan/

[5]唐瑞民，刘永东数字体育的发展及其人才的培养 [J]。广州体育学院学报, 2004 (6) : 97-99.

[6] 胡杨. 科技与健身的完美结合掀起数字潮流: 访北京体之杰体育用品有限公司董事长陆远大 [J]. 北京科 协, 2004(3):76-78.

\section{作者简介:}

田蕾蕾 (1982.5一), 女, 景德镇陶瓷大学讲师, 硕士, 研究方向: 数字体育

谢存军 (1978.8 一), 男, 景德镇学院讲师, 硕士, 研究方向: 体育艺术与审美

\section{References}

[1] (US) Negroponte wrote, Hu Yong, Fan Haiyan translation. Digital survival [M]. Hainan: Hainan Publishing House, 1997.

[2] Wang Li Chen. "Digital campus sports" construction [J]. Journal of Shenyang Physical Education Institute, 2003, 03: 20-22.

[3] http://www.apple.com/cn/watch/

[4] http://www.mi.com/shouhuan/

[5] Tang Ruimin, Liu Yongdong. The development of digital sports and personnel training [J].Guangzhou Institute of Physical Education, 2004 (6): 97-99.

[6] Hu Yang. The perfect combination of technology and fitness set off the digital trend: visit the Beijing TizhiJie Sporting Goods Co., Ltd. Chairman Lu Yuanda [Beijing], Beijing Science and Technology Association, 2004 (3): 76-78. 